\title{
Uso del material auténtico en las aulas virtuales para el aprendizaje de un idioma extranjero
}

Tolentino Quiñones, Hermis

Uso del material auténtico en las aulas virtuales para el aprendizaje de un idioma extranjero

Revista Educación, vol. 45, núm. 1, 2021

Universidad de Costa Rica, Costa Rica

Disponible en: http://www.redalyc.org/articulo.oa?id=44064134026

DOI: https://doi.org/10.15517/revedu.v45i1.42297

Esta obra está bajo una Licencia Creative Commons Atribución-NoComercial-SinDerivar 3.0 Internacional. 


\section{Uso del material auténtico en las aulas virtuales para el aprendizaje de un idioma extranjero}

\section{Use of authentic material in virtual classrooms for learning a foreign language}

Hermis Tolentino Quiñones

Istituto Italiano di Cultura di Lima, Perú

hermis_une@hotmail.com

iD http://orcid.org/0000-0002-9819-1655
DOI: https://doi.org/10.15517/revedu.v45i1.42297

Redalyc: http://www.redalyc.org/articulo.oa?id=44064134026

Recepción: 21 Junio 2020

Aprobación: 03 Agosto 2020

\section{ReSUMEN:}

El desarrollo de la tecnología ha permitido que actualmente haya cambios significativos en la educación, tanto es así que se habla de educación remota, poniéndose mayor atención a las aulas virtuales, al e-learning y al b-learning como espacios donde estudiantes y docentes pueden interactuar tal como se hace en las clases presenciales. Justamente, en este contexto, el material auténtico asume un rol importante en el aprendizaje de un idioma extranjero porque incentiva el desarrollo de la competencia lingüística, sociocultural y pragmática. En tal sentido, el presente ensayo tiene como objetivo reflexionar sobre el uso del material auténtico en las aulas virtuales de idiomas como lengua extranjera (LE) en la modalidad e-learning y b-learning. Por esta razón, en este ensayo se hizo una conceptualización de los argumentos presentados, identificando cualidades, ventajas, desafíos e implicaciones en la glotodidáctica. En este sentido, después de la revisión, análisis y reflexión de la bibliografía científica, se concluyó sobre la importancia del uso de las aulas virtuales y el material auténtico en el aprendizaje de un idioma extranjero y por tal motivo se le exhorta al profesorado de idiomas su uso en función de las realidades y necesidades del estudiantado.

Palabras Clave: Idioma extranjero, Material auténtico, Aulas virtuales, e-learning, b-learning.

\section{ABstract:}

The development of technology has allowed significant changes in education to take place today, so much so that, we talk about remote education, with more attention being paid to virtual classrooms, E-learning and B-learning as spaces where students and teachers can interact as it is done in the classroom. And this essay aims to reflect on the use of authentic material in virtual classrooms of languages as a foreign language (LE) in the E-learning and B-learning modality. In this context, authentic material plays an important role in the learning of a foreign language because it encourages the development of linguistic, sociocultural and pragmatic skills. In this sense, the objective of this essay is to reflect on the use of authentic material in virtual classrooms of languages as a foreign language (LE) in the E-learning and B-learning modality. For this reason, in this essay, a conceptualization of the presented arguments was made, identifying qualities, advantages, challenges, and implications in the glottodidactic. In this sense, after the reviewing, analyzing and reflecting about the theory it was concluded that the use of virtual classroom and authentic material in the learning of a foreign language is important and for that reason language teachers are encouraged to use them according to the realities and student needs.

KEYWORDS: Foreign languages, Authentic material, Virtual classrooms, E-learning, B-learning.

\section{Introducción}

La facilidad y la rapidez con la cual se puede tener acceso a la información a través de Internet y, al mismo tiempo, el desarrollo acelerado de las herramientas y los medios digitales, ha permitido que muchos de los conocimientos y las ciencias se desarrollen con mayor premura; así, por ejemplo, la educación ha sabido aprovechar los avances tecnológicos (Moreno y Aziz, 2019) para tratar de adaptar sus enfoques, métodos y técnicas de aprendizaje-enseñanza[1] a nuevas realidades para llegar a lo que hoy se conoce como educación remota. 
Es así como la educación remota se fortifica gracias al Internet, que supo desarrollar la comunicación bidireccional a través de medios escritos, verbales o visuales lo que ha permitido la libre transferencia de datos, audio, video, gráficos, etc. (García y Jáuregui, 2019). Por eso, existen en la actualidad (y más aún en la situación del COVID-19) muchas instituciones de educación superior (institutos, colegios, etc.) que brindan sus servicios educativos en espacios virtuales (Morado y Ocampo, 2018), principalmente en las modalidades del e-learning y del b-learning.

Además, la creación de espacios virtuales y el uso de Internet en favor de la educación son indispensables, porque, como manifiestan Rodríguez y Chávez (2020), el uso y manejo de las redes sociales en la actualidad es un estilo de vida de muchas personas y de generaciones venideras. Por eso, el sistema educativo y el profesorado deben considerar estos aspectos para adaptar la labor pedagógica a las necesidades del estudiantado y de la sociedad en general.

Entonces, el diseño de espacios virtuales para el proceso de aprendizaje-enseñanza (en la modalidad $e$ learning . b-learning) puede ser utilizado de dos formas: Primero, como simple repositorio donde acumular archivos y documentos, y sin ningún otro fin. Segundo, ser un espacio de interacción para la construcción de conocimientos con una participación responsable, reflexiva, cooperativa y creativa entre estudiantes y docentes (Morado y Ocampo, 2018).

Por ello, en el aprendizaje de idiomas, principalmente como lengua extranjera (LE), es importante implementar espacios virtuales para que haya una interacción continua, pues el aprendizaje de una lengua se fortifica cuando hay un acercamiento entre el alumnado y el idioma, el cual debe ser presentado en variados ámbitos y contextos. Y esto se puede hacer a través del material auténtico que sirve para presentar estructuras lingüísticas y también para conocer la realidad social y pragmática de la lengua en estudio (Carmona, Cruz y García, 2019).

Por lo expuesto, el presente ensayo tiene como objetivo reflexionar sobre el uso del material auténtico en las aulas virtuales de idiomas como lengua extranjera (LE) en la modalidad e-learning . b-learning. Para ello se hará un recuento y análisis de experiencias similares y la descripción y reflexión de algunos conceptos teóricos, divididos en dos ramas: el primero, sobre las aulas virtuales y la educación remota; el segundo, sobre el material auténtico y su importancia en la glotodidáctica.

Las experiencias y los estudios desarrollados subrayan que, cuando se diseñan cursos virtuales hay una responsabilidad entre el cuerpo docente, programadores y otros especialistas, y que la labor docente no podrá ser marginada ni reemplazada por otros. Sin embargo, el profesorado debe garantizar claridad sobre el desarrollo y uso de las aulas virtuales (Torres y Rodríguez, 2019) porque ellos trabajan directamente con el estudiantado y conocen las necesidades educativas.

Por consiguiente, el uso de la tecnología en el aprendizaje de idiomas debe incentivar en el estudiantado el desarrollo de su capacidad en la competencia comunicativa, crítica y reflexiva (Alarcón y Alarcón, 2018). Es así como, en una investigación sobre el desarrollo de las competencias sociolingüísticas y el uso de espacios virtuales en el aprendizaje del español como lengua extranjera, se determinó la importancia de activar el interés y la motivación de los y las discentes para profundizar los aspectos sociales y culturales a través del cortometraje como material auténtico, porque permite que el estudiantado tenga un acercamiento al uso pragmático de la lengua (Carmona et al., 2019).

\section{Argumentación}

Conceptos de aula virtual, e-learning y b-learning

En los últimos años, y más aún con la crisis del COVID-19, la educación remota, online y virtual se ha expandido y desarrollado a grandes pasos, ya sea en la educación formal o no formal (Moreno y Aziz, 2019). Sin embargo, no hay un concepto homogéneo de estos términos; lo que se ha tratado de hacer, para dar definiciones, es integrar algunos elementos referidos al uso de la tecnología, el tiempo que se dedica a cada forma de aprendizaje o los métodos de enseñanza (Costa, Celis, Castillo y Espinoza, 2019). 
Por ello, antes de dar una definición de aula virtual es necesario conceptualizar lo que se conoce como plataforma virtual o, como técnicamente es nominado, Learning Management System (LMS), es decir, Sistema de Manejo de Aprendizaje y de distribuido de forma sincrónica y asincrónica para poder interactuar a distancia (Vértiz, Pérez, Faustino, Vértiz y Alain, 2019). El aula virtual es más bien una concepción en el aspecto didáctico, pues se trata de transportar el concepto de aula física, donde se encontraban estudiantes, docentes, directivos, padres y madres de familia al ámbito virtual para interactuar como si lo hicieran comúnmente.

O sea, el aula virtual adquiere una mayor importancia cuando se deja de lado el concepto de plataforma virtual vista como simple repositorio para almacenar y transmitir información. El aula virtual se convierte en un espacio en el cual conviven prácticas y recursos que facilitan la comunicación, constituyéndose en una comunidad educativa donde se presta atención a las necesidades del estudiantado, el personal docente, los padres y las madres de familia y toda la comunidad educativa (Svensson, 2018).

Con respecto a la modalidad en cómo se puede aplicar las aulas virtuales, en la crisis de la pandemia COVID-19, muchas instituciones han adaptado sus metodologías al electronic learning (e-learning) y, seguramente, después de esta crisis, algunas instituciones educativas usarán el blended learning (b-learning), o sea, un aprendizaje semipresencial o mixto con el soporte de las TIC; esto con el objetivo de que la educación llegue a más personas y que se adapte a la realidad y a los tiempos actuales, porque, en la sociedad del conocimiento y en la era de la tecnología, el uso de los mass media (medios de comunicación masivos), del social network (red social), de los teléfonos inteligentes, de las tablets (tabletas), etc., es constante y cotidiano en la vida del estudiantado (Rodríguez y Chávez, 2020) y la educación no puede ser ajena a esta realidad.

Entonces, la educación en la modalidad electronic learning (e-learning) se refiere a clases $100 \%$ online, donde hace uso de los medios digitales (Moreno y Aziz, 2019) y las tecnologías electrónicas en todo el proceso educativo: en las estrategias de enseñanza y en los procesos de aprendizaje (García, 2020) y también en el proceso de evaluación. Por otro lado, la modalidad blended learning (b-learning) hace uso de la modalidad e-learning y las clases presenciales; generalmente, los encuentros presenciales permiten a la población estudiantil poner en práctica los aprendizajes adquiridos y reforzar los conocimientos con sus pares y despejar cualquier tipo de duda con el profesorado (Moreno y Aziz, 2019).

Caracteristicas de las aulas virtuales, e-learning y b-learning

La educación en general apunta a que el profesorado planifique, implemente y desarrolle el proceso de aprendizaje y enseñanza para lograr aprendizajes significativos; de igual manera, la educación remota comparte estos objetivos, solo que, en una educación no presencial, la comunicación debe ser aún más frecuente y fluida (García, 2020). Esto con el objetivo de alinearse a lo que manifiesta el constructivismo, al señalar que el mejor lugar donde aprender es aquel donde hay interacción activa entre docentes y discentes, y las actividades que se desarrollan le permiten al estudiantado elaborar sus propios conocimientos al interactuar entre pares (Arzeno, 2019).

Por ello, para Morado (2018, citado en Morado y Ocampo, 2018), los entornos virtuales (entendiéndose el e-learning y el b-learning) deben tener ciertas características para favorecer aprendizajes e interacciones eficaces: orientación para saber el uso de los diferentes elementos virtuales; desarrollar los contenidos que despierten la curiosidad del alumnado; un diseño visual homogéneo; amplitud de recursos para satisfacer las necesidades cognitivas, psicologías, comunicativas y creativas; actividades variadas y motivadoras; comunidad entre el grupo estudiantil y el profesorado a través de debates, foros, entre otros; y presencia docente para poder interactuar con el estudiantado, motivarlo, guiar el trabajo en conjunto, responder a dudas, etc.

Además, en un entorno de aprendizaje virtual es sumamente importante recordar que no se trata de un simple repositorio, sino que debe tener algunas características para lograr aprendizajes significativos y reflexivos; entre estas se tiene: A) El uso de los recursos audiovisuales y textuales presentes en Internet como herramientas y como medio de aprendizaje-enseñanza. B) La inclusión de contenidos complementarios: 
videos, lecturas, audios, ejercicios interactivos, links, etc. C) Como guía de contenidos y actividades: tareas, ejercicios, debates, entrega de tareas, etc. (Carmona et al., 2019).

En la misma línea de pensamiento, Lee et al. (2005 citado por Bachelor, 2019), sustentan que el aprendizaje en línea de un idioma extranjero como LE (en cualquiera de sus modalidades) debería considerar cuatro principios: 1) el suministro de actividades auténticas contextualizadas en situaciones lo más reales posibles, 2) el uso de gran cantidad de información con perspectivas diversas, 3) los espacios para la reflexión de modo práctico y 4) incentivar el trabajo cooperativo entre el estudiantado. A esto, el autor del presente ensayo agregaría otro principio: 5) autonomía, para que cada estudiante pueda ser autónomo en sus propios aprendizajes y logre desarrollar la metacognición.

\section{Ventajas y desafios del uso de las aulas virtuales, e-learning y b-learning}

La educación virtual presenta varias ventajas, desde el punto de vista práctico: A) Ahorro de costos en espacios físicos, desplazamiento, reutilización de materiales, etc. B) Mayor acceso a la educación y formación, ya que se puede llevar los cursos desde cualquier parte del mundo. C) Flexibilidad del proceso aprendizajeenseñanza, pues cada estudiante determina cuándo y dónde estudiar (Moreno y Aziz, 2019).

Además, desde el punto de vista didáctico, la educación remota, de acuerdo con Moreno y Aziz (2019), presenta cuatro ventajas, las cuales se describen a continuación:

A) Es personalizada, porque permite al estudiantado una mayor libertad para actuar de acuerdo con el propio ritmo y los propios intereses de aprendizaje, lo que permite así ir construyendo su propio aprendizaje. Cabe destacar que, cuando se dice libertad, no se refiere que el estudiante puede hacer y deshacer el proceso de aprendizaje-enseñanza. Para garantizar buenos resultados tendrá que confiar y ser guiado por su docente, quien es, no solo experto en el tema, sino también en la metodología y en la didáctica.

B) Incentiva el aprendizaje colaborativo, pues el aprendizaje es un proceso social. Trabajar en grupos permite expandir los conocimientos, confrontarse y construir, de forma cooperativa, los aprendizajes. Además, el trabajo cooperativo permite al aprendiz no sentirse aislado, sentimiento que se genera en las clases no presenciales (Costa et al., 2019). Pero, no se debe olvidar que el trabajo individual también es importante porque permite la metacognición. Obviamente, esto significa que el profesorado debe saber organizar las actividades didácticas y desarrollar en sus estudiantes las competencias comunicativas y socioafectivas adecuadas a cada situación educativa.

C) Permite el aprendizaje activo. La presentación de material interactivo: juegos, ejercicios y modelos de lengua online; permite que el estudiantado sea agente activo; asimismo, los ejercicios de simulación, análisis de casos, etc. incentivan un aprendizaje activo al interactuar con los pares.

D) Es un recurso multimedial por la presencia de material audiovisual, lecturas, links, blogs, foros, etc. Es decir, permite tener una red de recursos a disposición para poder lograr aprendizajes duraderos y reales de acuerdo con la propia necesidad educativa, comunicativa y lingüística. Aquí es importante que el personal docente sepa seleccionar los materiales y ordenarlos de acuerdo con las necesidades, de lo contrario la información sin filtro podría confundir al estudiantado.

Si bien es cierto que el aprendizaje-enseñanza remoto presenta grandes ventajas porque se adapta a las necesidades estudiantiles, también es cierto que hay grandes desafíos por superar. El primero está relacionado con la labor docente, porque se requiere una buena planificación tecnopedagógica (Costa et al., 2019) y una revisión de los aspectos didácticos y pedagógicos con el fin de humanizar los procesos formativos. Al mismo tiempo, se requiere una mayor creatividad e innovación (Rodríguez y Chávez, 2020) para lograr aprendizajes valiosos y profundos.

Otro desafío que es necesario superar es la falta de uniformidad en las clases que se brindan y en la calidad (Vásquez, 2018). Sobre todo, con la crisis del COVID-19, la gran mayoría de las instituciones educativas, públicas y privadas ha incursionado en clases virtuales sin tener la más mínima preparación. Es importante la alfabetización digital de su cuerpo docente (y de sus estudiantes) y el aspecto conceptual y metodológico para lograr procesos de interacción social en los ciberespacios (Rodríguez y Chávez, 2020). 
Por último, uno de los desafíos más grandes es la desigualdad educativa entre el área urbana y rural (Cuenca y Urrutia, 2019), por ejemplo, muchas personas no pueden tener acceso a Internet ni a los medios tecnológicos. Para ello, la política educativa debe cambiar, porque educación remota, a distancia, online, etc., no significa entregar laptops, sino, identificar el real problema, el cual se encuentra en la filosofía de la educación no presencial (García y Jáuregui, 2019), porque muchas instituciones educativas simplemente han trasladado la praxis presencial a la virtual, con errores y aciertos. Por ello, es primordial hacer cambios a nivel de enfoques, metodología, técnicas didácticas, instrumentos, etc.

\section{Concepto de material auténtico}

Begotti (s.f.) indica que el material auténtico pertenece a un tipo de material que es creado por un nativo sin ningún objetivo didáctico. Asimismo, en el Nozionario Di Glottodidattica de la Universidad Ca' FoscariVenezia (2020) se manifiesta que el material auténtico se refiere al uso de materiales que nacen con objetivos no didácticos, y entre ellos se encuentran los artículos periodísticos, los tiquetes de trenes, las películas, etc. Chamba y Gavilanes (2019) también coinciden en que el material auténtico no ha sido concebido para enseñar un idioma, sino que, fueron creados para comunicar significados. En otras palabras, el material auténtico se refiere a todo tipo de producción oral, escrita y audiovisual producida por un hablante nativo para otros hablantes nativos de la misma comunidad lingüística. Estos materiales auténticos pueden ser transmitidos por cualquier medio: radio, televisión, prensa, Internet, entre otros.

El término auténtico es usado por primera vez en 1975 por Wilkins y difundido ampliamente por el Método Directo (Begotti, s.f.). El enfoque comunicativo hace del material auténtico uno de sus componentes principales, porque en los últimos años el tránsito al extranjero se hace cada vez más común, el uso mundial de los medios de comunicación de masas permite transmitir información rápidamente y el desarrollo de la tecnología hace posible grabar comunicaciones reales (Nozionario Di Glottodidattica de la Universidad Ca' Foscari-Venezia, 2020) y todo esto permite que docentes y estudiantes de idiomas puedan hacer uso de una lengua más viva, real; es decir, más auténtica.

Pero, es muy importante entender la diferencia entre material auténtico y realia, este segundo término, de acuerdo con el Nozionario Di Glottodidattica de la Universidad Ca' Foscari (2020), se refiere a objetos y otros materiales auténticos. Nótese que se refiere principalmente a objetos (tiquetes, afiches, trípticos, etc.) que hacen parte del material auténtico, y esto último incluye audio, video, textos escritos, etc.; es decir, todo tipo de producción lingüística de un nativo para otros nativos de la misma comunidad.

\section{Tipología del material auténtico}

En el aprendizaje-enseñanza de un idioma extranjero es importante el uso de la tecnología, no solo en las clases remotas, sino también en las clases presenciales, porque ayuda a presentar el input, sobre todo si es multimedial, donde se integran imágenes, texto oral, texto escrito, ejercicios en línea, gramática de referencia, etc. (Balboni, 2002). Una mayor importancia tiene los materiales auténticos en el aprendizaje de idiomas. Estos materiales pueden ser clasificados de diversas formas, sin embargo, en este ensayo se ha querido seguir la clasificación que hace Begotti (s.f.), la cual se describe a continuación:

A) Material impreso: es el más difundido y usado en las clases de idiomas porque su fácil adaptabilidad. Entre estos se tiene: periódicos, revistas, afiches, mapas, libros, recetarios, etc. Estos materiales son los más usados porque pueden ser fotocopiados y adaptados con mayor facilidad respecto a otros tipos de materia; sin embargo, el uso excesivo podría ser menos motivante para los y las estudiantes.

B) Material video: se refiere a los programas de televisión, documentales, películas, entrevistas televisadas, incluso a grabaciones caseras que luego son transmitidas por las redes sociales. Es importante subrayar que, en realidad, este tipo de material es audiovisual y presenta un sinnúmero de ejemplos de lengua para el estudiantado. Además, permite no solo la comprensión del mensaje comunicativo a través del texto oral, sino que hace uso del lenguaje no verbal. También permite una mayor comprensión por la presencia de elementos paralingüísticos, entonación, velocidad del discurso, etc. 
C) Material audio: entre este tipo de material se encuentran la música-canción, los radioprogramas, la publicidad radiofónica, los audiolibros, etc. Generalmente, en las clases de idiomas se usan los diálogos, pero aquellos elaborados en un laboratorio, es decir no auténtico. El segundo material más usado son las canciones, pero muchas veces son empleadas solo para llenar vacíos o para relajar al estudiantado.

D) Material multimedial: por ser Internet un espacio donde es posible encontrar todo tipo de material, se considera material multimedial los audios, los videos y las lecturas. La información que se puede encontrar en Internet es un arma de doble filo, porque, por un lado, se puede encontrar gran cantidad de información y por otro, muchos presentan errores lingüísticos y no son clasificados en función del nivel lingüístico del alumnado y esto podría causar desmotivación y la activación del filtro afectivo.

Consideraciones en la elección y uso del material auténtico

Con el desarrollo de la tecnología e Internet, y el uso de la tecnología en el proceso de aprendizajeenseñanza de un idioma, se ha pasado de máquinas para aprender a máquinas para comunicar no a través de la explicación sino a través del uso del idioma que se estudia (Balboni, 2002). Asimismo, en el Marco Común Europeo de Referencias para las lenguas se propone un enfoque basado en la acción, donde la persona que aprende un idioma es considerada como un agente social cuyo objetivo es poder desenvolverse en la sociedad, valiéndose no solo de la competencia comunicativa, sino también, debe hacer uso de sus recursos cognitivos, emocionales y volitivos (Consejo de Europa, 2002).

Por ello, se debe incentivar en el estudiantado el desarrollo de las estrategias de comunicación a través de las cuatro habilidades de comprensión y producción escrita y oral, siguiendo los consejos descritos en el Marco Común Europeo, donde se dice que para aprender una lengua se debe leer textos escritos auténticos; escuchar la radio, las grabaciones; ver la televisión, películas; conversar con hablantes nativos; ser expuestos a enunciados auténticos, etc. (Consejo de Europa, 2002). Por esto, el personal docente debe saber elegir los materiales auténticos y, si es necesario, adaptarlos considerando las necesidades y realidades comunicativas del estudiantado.

Por consiguiente, en el momento de elegir el material auténtico se debe considerar: A) La infraestructura lingüística: la cual debe corresponder con el nivel del grupo de estudiantes y con los objetivos didácticos. Se debe analizar, asimismo, los elementos paratextuales, tales como: títulos, imágenes, cuadros, etc. B) Presencia de variedad lingüística: se podría encontrar en un material auténtico ejemplos de lengua de una determinada región, de un determinado registro, al mismo tiempo, se podría encontrar un lenguaje obsoleto y en tal caso el profesorado debe subrayar estos aspectos. C) Calidad del medio: especialmente si se trata de un material en video o audio; se debe comprobar la calidad sonora y visual. D) Presencia de elementos culturales: se debe verificar que estos elementos sean congruentes con lo que se desea que se aprenda y seleccionar bien el tema porque podrían ser considerados tabú por alguna parte del estudiantado (Begotti, s.f.).

Por último, es importante evidenciar que el material audiovisual integra varios códigos: visual, icónico, oral y sonoro, tal como sucede en la vida cotidiana, por ello, es considerado un material sumamente valioso en el aprendizaje de un idioma porque permite desarrollar la competencia lingüística (léxico, gramática, fonética, etc.), la competencia sociocultural y la competencia pragmática (Torresan, s.f.). Pero, su elección se debe hacer considerando el nivel lingüístico del estudiantado para mantener un input comprensible, una motivación alta y evitar la presencia del filtro afectivo.

$\mathrm{Al}$ ser el objetivo de este ensayo reflexionar sobre el uso del material auténtico y las aulas virtuales en el aprendizaje de idiomas, se desarrolló la búsqueda exhaustiva de la literatura científica, se analizaron experiencias similares y se sustentaron argumentos teóricos divididos en dos campos: el primero, relacionado con las aulas virtuales, el e-learning y el b-learning; se conceptualizaron estas terminologías y se expusieron las características, las ventajas y los desafíos por enfrentar. El segundo tema desarrollado es el material auténtico, se describieron la tipología y las implicaciones didácticas al momento de seleccionarlas para su uso didáctico.

La metodología de trabajo empleada en este ensayo permitió reforzar la hipótesis sobre la utilidad que tiene el material auténtico en las aulas virtuales para el aprendizaje y la enseñanza de un idioma extranjero 
planteamiento que coincide con Carmona et al. (2019), al sustentar la importancia del uso de las plataformas virtuales y el uso de cortometrajes como material auténtico para desarrollar las competencias socioculturales en el aprendizaje de un idioma extranjero. Sin embargo, se debe subrayar que cualquier tipo de material auténtico audiovisual debe ser original, es decir, no se debe usar aquellos doblados porque el lenguaje no verbal podría no coincidir. Así, por ejemplo, la población gesticula mucho al momento de hablar, mientras que la población oriental no, y una película china, por ejemplo, doblada en italiano no tendría efectos didácticos positivos.

Así mismo, el marco teórico presentado sobre las aulas virtuales guarda relación con lo que sustentan Torres y Rodríguez (2019), quienes manifiestan que el profesorado debe garantizar claridad sobre el desarrollo y el uso de las aulas virtuales, porque trabaja directamente con la población estudiantil y son quienes deciden qué tipo de material utilizar, qué estrategias emplear y la modalidad de trabajo: individualmente, en parejas o en grupos.

\section{Conclusiones}

De acuerdo con la bibliografía presentada, la revisión, el análisis y la interpretación de los conceptos, se concluye que, en la actualidad, en la educación remota es muy importante el uso de las aulas virtuales, ya sea en la modalidad e-learning o b-learning, para incentivar la interacción entre estudiantes y docentes de idiomas, como lengua extranjera (LE) o como segunda lengua (L2).

Así mismo, se concluye sobre la importancia del uso del material auténtico en todas sus variedades a través del aula virtual en el aprendizaje de un idioma extranjero. Y que entre los materiales más completos se tiene al audiovisual, porque permite desarrollar la competencia lingüística, sociolingüística y pragmática de la lengua. También es altamente motivante y desarrolla la comunicación verbal y no verbal.

También se concluye que, como existe abundancia y variedad de material auténtico digital en Internet, el profesorado debería saber seleccionarlos en función de las necesidades y las realidades comunicativas del estudiantado.

Por último, se concluye que el uso de los materiales auténticos a través de las aulas virtuales en la educación remota de idiomas es necesario para las generaciones del presente y del futuro y se hace una recomendación imperativa para su uso.

\section{ReFERENCIAS}

Alarcón, H. y Alarcón, M. (2018). E-learning y la gestión de los aprendizajes en estudiantes de 4to año de educación básica en lima, Perú. Investigación y Postgrado, 33(2), 155-165. Recuperado de https://dialnet.unirioja.es/serv let/articulo?codigo $=6736281$

Arzeno, A. (2019). El uso de la plataforma moodle en el rendimiento académico de lenguaje II de estudiantes universitarios, 2019. (Tesis maestría). Universidad San Martín de Porres, Perú. Recuperado de http://repositorio.usmp.edu.p e/handle/usmp/5917

Bachelor, J. (2019). El aula presencial, semipresencial, virtual e invertida: Un estudio comparativo de métodos didácticos en la enseñanza de L2. Revista Educación, 43(2), 20. doi: https://doi.org/10.15517/revedu.v43i2.3 4014

Balboni, P. (2002). Le sfide di Babele. Italia: UTET Università

Begotti, P. (s.f.).Didattizzazione dimateriali autenticie analisi dei manuali di italiano perstranieri. Venezia: Ca' Foscari - FILIM. Recuperado de https://www.itals.it/sites/default/files/Filim_didattizzazione_analisi_teoria.pdf

Carmona, M., Cruz, V. y García, L. (2019). Desarrollo de competencias sociolingüísticas e interculturales en ELE: propuesta didáctica con blended learning. Revista Comunicación, 28(1-2019), 16-30. doi: https://doi.org/10. $18845 /$ rc.v28i1-2019.4442 
Consejo de Europa. (2002). Marco Común Europeo De Referencia Para Las Lenguas: aprendizaje, enseñanza y evaluación. Centro Virtual Cervantes. Recuperado de http://cvc.cervantes.es/ensenanza/biblioteca_ele/marco/ default.htm

Costa, P., Celis, K., Castillo, N. y Espinoza, G. (2019). Análisis de la implementación institucional de la modalidad blearning en carreras de pregrado de tres universidades chilenas. Calidad en la educación, (50), 216-255. doi: htt ps://dx.doi.org/10.31619/caledu.n50.722

Chamba, M., y Gavilanes, C. (2019). Authentic audio-visual material in the development of oral fluency in university intermediate english students. Literatura y lingüistica, (39), 199-223. doi: https://dx.doi.org/10.29344/07176 $21 x .39 .2011$

Cuenca, R. y Urrutia, C. (2019). Explorando las brechas de desigualdad educativa en el Perú. Revista mexicana de investigación educativa, 24(81), 431-461. Recuperado de https://bit.ly/3mJt0LY

García, J. y Jáuregui, P. (2019). Educación a distancia y mundos virtuales. Revista de Investigación Universidad Tecnológica de Pereira, 1(2), 163-177. doi: http://dx.doi.org/10.22517/25393812.22051

García, L. (2020). Bosque semántico: ¿educación/enseñanza/aprendizaje a distancia, virtual, en línea, digital, eLearning...? RIED. Revista Iberoamericana de Educación a Distancia, 23(1), 9-28. doi: https://doi.org/10.594 4/ried.23.1.25495

Morado, M. y Ocampo, S. (2018). Una experiencia de acompañamiento tecno-pedagógico para la construcción de entornos virtuales de aprendizaje en educación superior. Revista Educación, 43(1), 43-60. doi: https://doi.org $/ 10.15517 /$ revedu.v43i1.28457

Moreno, A. y Aziz, C. (2019). Formación continua online: Aprendizajes para el futuro de los líderes educativos. Informe Técnico $N^{o}$ 7. Chile: LIDERES EDUCATIVOS, Centro de Liderazgo para la Mejora Escolar. Recuperado de https://bit.ly/3cuNK5r

Nozionario Di Glottodidattica de la Universidad Ca' Foscari-Venezia. (2020). Definiciones de terminaologia didáctica. Università Ca' Foscari di Venezia. Recuperado de https://www.itals.it/nozion/noziof.htm

Rodríguez, A. y Chávez, E. (2020). Cibernética educativa, actores y contextos en los sistemas de educación superior a distancia. Sophia-Colección De Filosofía De La Educación, (28), 117-137. doi: https://doi.org/10.17163/sop h.n28.2020.04

Svensson, V. (2018). Análisis de portadas de aulas virtuales. Ciencia, Docencia y Tecnología, 29(56), 156-185. doi: h ttps://doi.org/10.33255/2956/329

Torres, C. y Rodríguez, J. (2019). Los entornos de aprendizaje inmersivo y la enseñanza a ciber-generaciones. Educação e Pesquisa, 45, 1-20. doi:https://doi.org/10.1590/s1678-4634201945187369

Torresan, P. (s.f). Tecnologie per l'apprendimento dell'italiano. Venezia: Ca' Foscari-FILIM. Recuperado de https:// www.itals.it/sites/default/files/Filim_tecnologie_teoria.pdf

Vásquez, L. (2018). Didáctica para la educación no presencial en adultos. Espirales Educativas, 3(2), 54-67. Recuperado de https://emeega.files.wordpress.com/2019/01/espirales_educativas.pdf

Vértiz, R., Pérez, S., Faustino, M., Vértiz, J. y Alain, L. (2019). Tecnología de la Información y Comunicación en estudiantes del nivel primario en el marco de la educación inclusiva en un Centro de Educación Básica Especial. Propósitos y Representaciones, 7(1), 146-164. doi: http://dx.doi.org/10.20511/pyr2019.v7n1.266

\section{Notas}

[1] El ser humano es el fin de la educación, por ello, se debería hablar de aprendizaje-enseñanza.

\section{BY-NC-ND}

